\title{
Reproductive age mortality survey: a tool to determine level of underreporting of maternal mortality
}

\author{
Sonika Badalia1 ${ }^{1}$ Anupam Parashar ${ }^{2 *}$, D. S. Dhadwal ${ }^{2}$, Deepak Sharma ${ }^{3}$
}

\begin{abstract}
${ }^{1}$ Senior Project Officer, Health System Strengthening, UNDP, Himachal Pradesh, India
${ }^{2}$ Department of Community Medicine, Indira Gandhi Medical College, Shimla, Himachal Pradesh, India

${ }^{3}$ Department of Community Medicine, Government Medical College and Hospital, Chandigarh, India
\end{abstract}

Received: 06 November 2017

Accepted: 02 December 2017

\section{*Correspondence:}

Dr. Anupam Parashar,

E-mail: anupamvikrant@yahoo.co.in

Copyright: $\odot$ the author(s), publisher and licensee Medip Academy. This is an open-access article distributed under the terms of the Creative Commons Attribution Non-Commercial License, which permits unrestricted non-commercial use, distribution, and reproduction in any medium, provided the original work is properly cited.

\begin{abstract}
Background: For achieving sustainable development goals, tracking all maternal deaths without missing any is a crucial step. Underreporting of maternal deaths is an issue especially in developing countries like India. Objectives of this study were to estimate maternal deaths using Reproductive Age Mortality Survey (RAMOS) methodology in a district of North India and to study medical causes responsible for maternal deaths.

Methods: A Reproductive Age Mortality Study (RAMOS) was carried out in a district of North India for the period 2009 to 2012. Records of female deaths in the reproductive age group were reviewed using multiple sources and verbal autopsy was conducted. Maternal deaths thus identified were compared with the officially reported maternal deaths for the same period for finding out the under reporting. Verbal autopsy was conducted to find out causes of maternal deaths.

Results: A total of 16 women were identified as maternal deaths using RAMOS methodology. The officially reported maternal deaths were 8 , thus under reporting of $100 \%$. The 16 maternal deaths consisted of $14(87.5 \%)$ direct maternal deaths and $2(12.5 \%)$ indirect maternal deaths. One or the other form of delay was recognized in 8 of 16 maternal deaths. There was delay in recognizing the warning signs for urgent health care requirement (first delay) in 4 of 16 maternal deaths. There was delay on part of health facility to diagnose the complication and act appropriately (third delay) in 4 cases.

Conclusions: It is concluded that the reporting system for maternal deaths need to be strengthened. Health workers should be adequately trained on reporting maternal death cases. On this foundation verbal autopsy should be conducted for all the reported deaths of women of reproductive age group to know the circumstances leading to maternal deaths.
\end{abstract}

Keywords: Maternal deaths, Reproductive age mortality survey, Underreporting

\section{INTRODUCTION}

An estimated 830 women die from preventable causes related to pregnancy and childbirth every day, $99 \%$ of them in developing countries. As a part of the sustainable development goals agenda, the target is to reduce the global maternal mortality ratio to less than 70 per 100
000 live birth. To accomplish this, it is essential to track all maternal deaths and thus guide actions to prevent any future deaths ${ }^{1}$.

Worldwide, different approaches are used to ascertain maternal deaths, such as community-based verbal autopsies, facility based maternal deaths reviews and 
reproductive age mortality studies. RAMOS provides an estimate of the proportion of maternal deaths among female deaths in the reproductive age group. This reliable method has been used successfully in different counties around the world like Egypt, Honduras and Jamaica and Afghanistan. ${ }^{2,3}$

Tracking all maternal deaths without missing any helps in assessing the exact magnitude of maternal mortality and thus in turn, derives policy maker's response it deserves. With this background the study was done with the aim to conduct recording of maternal deaths using Reproductive Age Mortality Survey (RAMOS) methodology in a district of North India and to identify the degree of underreporting for maternal deaths.

\section{METHODS}

This retrospective study was done over a period of one year i.e. September 2012 to August 2013. The study was conducted in Hamirpur district of Himachal Pradesh. Around $93 \%$ of the population resides in rural area and around $20 \%$ of the families live below poverty line. The study population was the institutional maternal deaths and deaths of women in reproductive age group (15-49 years) during the year 2009-2012.

Reproductive Age Group Mortality Survey (RAMOS) was conducted to find out the maternal deaths. In addition to this, institutional maternal mortality data (public and private hospitals) and snowball sampling was used to find out the maternal deaths. Link workers of the respective areas were also consulted to find any additional cases. For RAMOS, women in reproductive age group mortality data were collected from civil registration system and from death registers of all the public health care centres (health subcentres to district hospital).

Based on the above-mentioned methodology verbal autopsy was conducted for all women deaths in the reproductive age group as well as reported maternal deaths. Prior written informed consent was obtained from the respondents. Primary respondent was taken as the person present at time of death, preferably the husband (or father) or mother in law (or mother). In cases of unclear reporting, secondary respondents were interviewed which comprised of, neighbors, health worker or TBA attending to the deceased. Hospital records, if available were noted. To distinguish maternal from non-maternal deaths we used WHO developed 'International standard verbal autopsy questionnaire 3; death of a person aged 15 years and above'. In cases of established maternal deaths cause of death was ascertained using pre-structured (combined) questionnaire designed by ICMR for estimation of maternal mortality ratio in Indian states. ${ }^{4}$ Gynecologists' opinion was taken in cases in which cause of death was unclear via verbal autopsy and medical records not available. Data was analyzed using the SPSS software for windows.
For calculating under reporting, the maternal deaths identified through RAMOS were compared with those reported and documented in officially reported data. The officially reported maternal deaths data was collected from all public and private hospitals of the district conducting deliveries. The study protocol was approved by the institutional ethics committee. Permission to conduct the study was obtained from the Mission Director, National Rural Health Mission, Himachal Pradesh.

\section{RESULTS}

The total number of female deaths in their reproductive age (15-49 years) obtained together from vital registration system and health records during reference period, i.e. from April 2009 to March 2012 in district Hamirpur was 398. Among those, a total of 16 women died while pregnant or within 42 days of termination of a pregnancy. The official health records of the district reported eight maternal deaths, thus under reporting of $100 \%$ (Table 1).

Table 1: Identified maternal deaths in Hamirpur district of Himachal Pradesh.

\begin{tabular}{|l|l|}
\hline $\begin{array}{l}\text { Identified maternal deaths through } \\
\text { RAMOS }\end{array}$ & $\begin{array}{l}\text { Number of } \\
\text { maternal deaths }\end{array}$ \\
\hline Officially reported Deaths & 16 \\
\hline Underreported & 8 \\
\hline
\end{tabular}

During this study no additional maternal death was found using snowballing technique. The deaths that were reported by applying the snowballing method had already been recorded by RAMOS.

One case out of 16 maternal deaths did not seek antenatal care. The remaining 15 cases $(93.75 \%)$ were registered with health system during antenatal period. The 16 maternal deaths consisted of 14 direct maternal deaths $(87.5 \%)$ and 2 indirect maternal deaths (12.5\%). The majority of maternal deaths occurred in the postnatal period. The most frequent causes of maternal deaths were postpartum hemorrhage $(6 ; 37.5 \%)$ followed by pregnancy induced hypertension $(2 ; 12.5 \%)$, amniotic fluid embolism $(2 ; 12.5 \%)$ and post anesthetic complication $(1 ; 6.5 \%)$. The remaining five maternal deaths occurred from other causes which included fever (3), spontaneous uterine inversion followed by neurogenic shock (1), and hemorrhage from mismanaged dosage of anticoagulant therapy following deep vein thrombosis in postpartum period (1) (Table 2). $31.25 \%$ of maternal deaths occurred before age of 25 years, $56.25 \%$ occurred between age $25-29$ years and $6.25 \%$ each in the age 30-34 years and 35-39 years. Out of all the maternal deaths, 4 maternal deaths occurred at parity one, 5 at second parity, 4 at parity three and 1 in the postnatal 
period at parity four; 2 women died during first pregnancy (nulliparous).

Table 2: Causes of maternal deaths.

\begin{tabular}{|lll|}
\hline & $\mathrm{N}=16$ & $\%$ \\
\hline Direct cause & & \\
\hline Postpartum hemorrhage & 6 & $37.5 \%$ \\
\hline Pregnancy Induced Hypertension & 2 & $12.5 \%$ \\
\hline Amniotic Fluid Embolism & 2 & $12.5 \%$ \\
\hline Fever & 3 & $18.8 \%$ \\
\hline Spontaneous uterine inversion & 1 & $6.3 \%$ \\
\hline Indirect cause & & \\
\hline $\begin{array}{l}\text { Hemorrhage from mismanaged } \\
\text { dosage of anticoagulant therapy }\end{array}$ & 1 & $6.3 \%$ \\
\hline Post anaesthesia complication & 1 & $6.3 \%$ \\
\hline
\end{tabular}

10 of 16 maternal deaths cases underwent institutional delivery. One of them gave birth at home. The remaining $5(31.25 \%)$ women died before giving birth. Of these 5 deaths, 3 occurred before 28 weeks period of gestation (POG), 1at 33 weeks POG and 1died at full term.

$12(75 \%)$ maternal deaths occurred in the hospital. The balance $4(25 \%)$ deaths occurred on way to subsequent hospital. None of the recorded maternal deaths occurred at home. In most instances women who die in childbirth are found to have experienced at least one of the following three delays: The 'first delay' is in deciding to seek care for an obstetric complication. The 'second delay' occurs after the decision to seek care has been taken. There is delay in coordinating the method of transportation, resulting in a delay in reaching the care facility. The 'third delay' occurs in obtaining care at the facility. One or the other form of delay was recognized in 8 of 16 maternal deaths. There was delay in recognizing the warning signs for urgent health care requirement (first delay) in 4 of 16 maternal deaths. There was delay on part of health facility to diagnose the complication and act appropriately (third delay) in 4 cases.

$12.5 \%$ of the maternal death cases had called 108 ambulance services for primary shifting from home to hospital.

\section{DISCUSSION}

Present study highlights underreporting of maternal deaths. The present study has brought about twice the number of maternal deaths over the reference period of three years than that reported by the district health records (16 against 8 ). In the present study we found that maternal mortality was underestimated by hundred percent. This highlights the importance of tracking maternal deaths using multiple methods. Underreporting of maternal deaths has been reported in surveys done in different studies across the globe. A study done by Zakariah in Ghana reported under reporting by $44 \% .^{5}$ Persistent underreporting of maternal deaths has been mentioned in studies done by Bouvier-Colle in France and Schuitemaker in Netherlands. ${ }^{6,7}$

It was found that most of the maternal deaths (15 out of total 16 maternal deaths) were registered with health system during antenatal period, but the official health system only reported half of it. This shows the inadequacy and ineffectiveness of our health system in tracking the maternal deaths for the registered antenatal cases. The health workers have not reported all the maternal deaths occurring in their areas. There is still confusion regarding reporting of referred maternal deaths that occur during transportation or on reaching the referral health unit.

In relation to parity, our maternal death profile showed maximum deaths in second pregnancy. In contrast to this finding a study done by Das in India observed that maximum deaths were in primigravidas as compared to multigravidas. ${ }^{8}$ In the present study, maximum deaths occurred in the post-partum period. Similar results have also been reported by other studies. ${ }^{9,10}$

The high numbers of deaths in post-partum period indicate the need for vigilance in post-partum period and prompt action in case any problem is identified. We found that the most common cause of death was hemorrhage. In studies from India by Purandre et al and Priya et al hemorrhage was the leading cause of maternal mortality $35.05 \%$ and $70.83 \%$, respectively. ${ }^{10,11}$ However studies done by Singh et al and Paul et al, listed hypertensive disorders of pregnancy the major cause of maternal mortality. ${ }^{12,13}$

Post-natal care still remains an unattended area. The majority of maternal deaths occurring in the postnatal period raise doubts on our postnatal services. The district has achieved high institutional delivery rate. As per verbal autopsy, discharge from hospital was as per norms, i.e. after two days following normal vaginal delivery and five days after caesarean delivery. In spite of this majority of maternal deaths in postnatal period is the real cause of concern raising doubts on home based care.

A free ambulance services with public private partnership under the name of 108 was started in Himachal Pradesh in the year 2010. To avail the ambulance services, one has to dial 108 from any phone. Present study found out that only $12.5 \%$ of the maternal death cases had called this ambulance for primary shifting from home to hospital. The reason can be the new service developed its credibility over a period of time. In majority of the cases private taxi was used to shift the patient to the hospital.

Present study pointed out 50\% maternal deaths occurring due to one or the other of the three delays.

The identified delays in maternal deaths were delay in ability to decide when to seek care in an obstetric emergency (first delay) and delay in receiving medical 
care at the level of primary health facility (third delay). The first delay may be because of inability to identify the danger signs of an impending obstetric complication within time. Delay in receiving care at the facility may be due to absence of health staff, late referral to higher health facility and may be due to unavailability of emergency obstetric services at the facility. In a study conducted in Indonesia, using verbal autopsy method it was found that delays in seeking, reaching and receiving care occurred in $70 \%$ of the maternal death cases. First delay was reported in $45 \%$ cases, second delay in $66 \%$ and third delay in $44 \%$ cases. $^{14}$

In the present study, avoiding delay in recognizing complications and providing adequate maternity care could have saved eight mothers out of sixteen from dying. This may be due to the fact that currently in reproductive and child health services, we are focusing more on indicators for improving numbers in terms of antenatal registrations, TT administration, IFA tablet distribution than improving quality of routine antenatal care, in terms of preventing, diagnosing, or treating complications. ${ }^{15}$

The study has some limitations. There is a possibility of missing some teenage maternal deaths which may have gone unreported due to the social reasons. There is possibility of missing some early maternal deaths during abortion again due to social factors.

\section{CONCLUSION}

It is concluded that the reporting system for maternal deaths need to be strengthened. Health workers should be adequately trained on reporting maternal death cases whether occurring at home, institution or on the way to subsequent hospital. This can be achieved by training them in monthly meetings. The link workers like Anganwadi workers, ASHA and Panchayat Chowkidars can be encouraged via monetary incentives to report all female deaths in the reproductive age group (15-49 years) on monthly basis to the health workers of their respective area. On this foundation verbal autopsy should be conducted for all the reported cases by trained health workers/medical officers.

A major step in the state for preventing maternal deaths is that maternal death review has been started in the state since 2015. Also, $\mathrm{RMNCH}+\mathrm{A}$ guidelines along with supportive supervision are now being fully adhered to in the state to decrease institutional based maternal deaths and to recognise complications in time so that timely referrals can be made.

Funding: No funding sources Conflict of interest: None declared
Ethical approval: The study was approved by the Institutional Ethics Committee

\section{REFERENCES}

1. World Health Organization: Trends in Maternal Mortality: 1990 to 2008 Estimates developed by WHO, UNICEF, UNFPA and The World Bank. Geneva. 2010

2. RAMOS. Available at http://www.who.int/reproductivehealth/topics/monitorin g/measuring_mmr.pdf. Accessed 24 ${ }^{\text {th }}$ April 2016.

3. Abouzahr C. In: Critical issues in safe motherhood. Geneva, WHO; 1999:110-134.

4. Estimates of maternal mortality ratios in India and its states: a pilot study. Available at www.icmr.nic.in/final/Final\%20Pilot\%20Report.pdf Cited 17 February 2016.

5. Zakariah AY, Alexander S, Roosmalen JV. Reproductive age mortality survey (RAMOS) in Accra, Ghana. Reproductive Health. 2009;6:7.

6. Bouvier-Colle MH, Varnoux N, Costes P, Hatton F. Reasons for the underreporting of maternal mortality in France, as indicated by a survey of all deaths among women of childbearing age. Int $\mathbf{J}$ Epidemiol. 1991;20:717-21.

7. Schuitemaker NWE, van Roosmalen J, Dekker GA, van Dongen PWJ, van Geijn HP, Bennebroek Gravenhorst $\mathrm{J}$ : Underreporting of maternal mortality in The Netherlands. Obstet Gynecol. 1997;90:78-82.

8. Das R, Biswas S, Mukherjee A. Maternal mortality at a teaching hospital of rural India: a retrospective study. Int J Biomed Advance Res. 2014;5:114-7.

9. Dogra P, Gupta KB. A study of maternal mortality at a tertiary institute. Obs Gynae Today. 2009;115:58-60.

10. Purandare N, Singh A, Upadhyae S, Saraogi RM. Maternal mortality at a referral centre: a five year study. J Obstet Gynaecol India. 2007;57:248-50.

11. Priya N, Verma A, Verma S. Maternal mortality: ten years retrospective study. J Med Educ Res. 2010;12:134-6.

12. Singh R, Sinha N, Bhattacharyya K, Ram R. Pattern of maternal mortality in a tertiary care hospital of Patna, Bihar. Indian J Community Med. 2009;34:73-4.

13. Paul B, Mohpatra B, Kar K. Maternal deaths in a tertiary health care centre of Odisha: an in-depth study supplemented by verbal autopsy. Indian J Community Med. 2011;36:213-6.

14. Qomariyah SN, Ambruoso L. Indonesia: extending verbal autopsy to examine social and medical causes of maternal mortality. Impact briefing note: November 2008. Avalaible at http://r4d.dfid.gov.uk/PDF/Outputs/Immpact/Indonesia BriefingNote.pdf. Cited 5th May 2016.

15. McDonagh $\mathrm{M}$. Is antenatal care effective in reducing maternal morbidity and mortality? Health Policy Plan. 1996;11(1):1-15.

Cite this article as: Badalia S, Parashar A, Dhadwal DS, Sharma D. Reproductive age mortality survey: a tool to determine level of underreporting of maternal mortality. Int J Reprod Contracept Obstet Gynecol 2018;7:220-3. 